\title{
Vitrification, in vitro fertilization, and development of Atg7 deficient mouse oocytes
}

\author{
Soyoung Bang ${ }^{1 *}$, Geun-Kyung Lee ${ }^{1 *}$, Hyejin Shin ${ }^{1}$, Chang Suk Suh ${ }^{2}$, Hyunjung Jade Lim ${ }^{1,3}$ \\ 'Department of Biomedical Science and Technology, Institute of Biomedical Science and Technology, Konkuk University, Seoul; ${ }^{2}$ Department of \\ Obstetrics and Gynecology, Seoul National University Bundang Hospital, Seongnam; ${ }^{3}$ Department of Veterinary Medicine, Konkuk University, Seoul, \\ Korea
}

Objective: Autophagy contributes to the clearance and recycling of macromolecules and organelles in response to stress. We previously reported that vitrified mouse oocytes show acute increases in autophagy during warming. Herein, we investigate the potential role of Atg 7 in oocyte vitrification by using an oocyte-specific deletion model of the Atg7 gene, a crucial upstream gene in the autophagic pathway.

Methods: Oocyte-specific Atg7 deficient mice were generated by crossing Atg7 floxed mice and Zp3-Cre transgenic mice. The oocytes were vitrified-warmed and then subjected to in vitro fertilization and development. The rates of survival, fertilization, and development were assessed in the Atg7 deficient oocytes in comparison with the wildtype oocytes. Light chain 3 (LC3) immunofluorescence staining was performed to determine whether this method effectively evaluates the autophagy status of oocytes.

Results: The survival rate of vitrified-warmed $A \operatorname{tg}{ }^{f / f} ; \mathrm{Zp3}-\mathrm{Cre}\left(A \operatorname{tg} 7^{\mathrm{d} / \mathrm{d}}\right)$ metaphase II (MII) oocytes was not significantly different from that of the wildtype (Atg $7^{t / f}$ ) oocytes. Fertilization and development in the Atg $7^{d / d}$ oocytes were significantly lower than the Atg $7^{t / f}$ oocytes, comparable to the Atg $5^{d / d}$ oocytes previously described. Notably, the developmental rate improved slightly in vitrified-warmed Atg $7^{d / d}$ Mll oocytes when compared to fresh Atg $7^{d / d}$ oocytes. LC3 immunofluorescence staining showed that this method can be reliably used to assess autophagic activation in oocytes.

Conclusion: We confirmed that the LC3-positive signal is nearly absent in Atg $7^{d / d}$ oocytes. While autophagy is induced during the warming process after vitrification of MII oocytes, the Atg7 gene is not essential for survival of vitrified-warmed oocytes. Thus, induction of autophagy during warming of vitrified MII oocytes seems to be a natural response to manage cold or other cellular stresses.

Keywords: Atg7; Autophagy; Oocytes; Vitrification

\section{Introduction}

Macroautophagy (referred to hereafter as autophagy) is an intracellular catabolic mechanism that degrades old or dysfunctional cellular

Received: Jan 11, 2016 · Revised: Feb 6, 2016 · Accepted: Feb 10, 2016 Corresponding author: Hyunjung Jade Lim

Department of Veterinary Medicine, Konkuk University, 120 Neungdong-ro, Gwangjin-gu, Seoul 05029, Korea

Tel: +82-2-450-4087 Fax: +82-2-2030-7809 E-mail: hlim@konkuk.ac.kr

*These authors contributed equally to this study.

* This study was supported by a grant from the Korea Health Technology R\&D Project, Ministry of Health \& Welfare, Korea (H12C0055).

This is an Open Access article distributed under the terms of the Creative Commons Attribution Non-Commercial License (http://creativecommons.org/licenses/by-nc/3.0/) which permits unrestricted non-commercial use, distribution, and reproduction in any medium, provided the original work is properly cited. components through lysosomes [1]. Catabolic products are then recycled to generate new membranes and macromolecules to maintain normal cell function [2]. This process is thought to occur at a basal level in all cells while certain signals upregulate or suppress this process. Many inducers of autophagy have been identified, including starvation, deprivation of growth factors or hormones, and temperature stress [2-4]. The process mostly relies on a well-characterized multistep pathway involving proteins encoded by various autophagy genes (Atg). Among these, Atg5 and Atg7 are widely recognized as upstream effectors whose actions are required for autophagosome formation. Atg 5 forms a complex with Atg12, and this complex acts as an E2-like enzyme mediating the lipidation of Atg8 (light chain 3, LC3) protein. Lipidated LC3, LC3-II, is the phosphoethanolamine conjugated form and is incorporated into the autophagosomal membrane. 
Lipidation of LC3, i.e., the conversion of LC3-I to LC3-II, is often used as a marker of autophagic flux [1]. Atg7 is an E1-like enzyme that conjugates with LC3 and Atg12 during the biogenesis of autophagosomes. Therefore, it sits at the upstream of autophagic activation $[5,6]$. The mouse model of floxed Atg7 is now widely used to investigate the effects of defective autophagy in various tissues and organs $[7,8]$. Zona pellucida protein 3 (Zp3) gene encodes an oocyte-specific protein expressed during oogenesis [9]. The Zp3-Cre transgenic mouse model is used to drive deletion of the floxed gene in oocytes specifically [10].

We have previously showed that mouse oocytes show a heightened autophagic response during warming after vitrification [11]. Along with several ultrastructural and cellular changes [12,13], autophagy is recognized as a cellular response to various stresses to oocytes and embryos $[11,14]$. However, the role of autophagic activation during warming is unclear, as vitrified-warmed oocytes are generally considered normal with respect to rates of survival, fertilization, and subsequent development in mice [15]. Thus, autophagic activation observed during warming could be a cell response associated with restoration of potential cellular damage done by temperature stress. Alternatively, autophagy could be activated as a natural response to cold stress but may not have a necessary function for survival. Thus, it remains to be determined whether a complete absence of autophagy has any effect on the survival, fertilization, or development of vitrified-warmed oocytes. In this work, we aim to address two aspects of autophagy in mouse oocytes: first, the consequences of a complete absence of Atg7, a seminal gene in autophagy, in vitrified-warmed oocytes, and second, assessment of LC3 immunofluorescence staining as a reliable method of monitoring autophagy in oocytes.

\section{Methods}

\section{Mice}

All mice were maintained in strict accordance with the policies of the Konkuk University Institutional Animal Care and Use Committee (IACUC). This study was approved by the Konkuk University IACUC (approval no. KU14099). Atg7 floxed mice (Atg7f/f mice, C57BL/6) [7] were obtained from the RIKEN BioResource Center (Ibaraki, Japan). Zp3-Cre transgenic mice (C57BL/6) were purchased from Jackson Laboratory (Bar Harbor, ME, USA). To produce the Atg ${ }^{f / f} ; \mathrm{Zp} 3-\mathrm{Cre}$ mice, Atg $^{f / /}$ female mice were mated with Atg $^{f / /} ; Z \mathrm{Zp} 3-C$ re male mice, and the litters were selected as either experimental or control depending on the genotype (Table 1). To improve reproductive performance, the mice were crossed onto an ICR background. Deletion of the Atg7 gene in the oocytes of the Atg ${ }^{f / f} ; \mathrm{Zp3}-C r e$ mice was confirmed by reverse transcription polymerase chain reaction (RT-PCR) (Table 1).

\section{Collection of matured oocytes}

Atg $7^{f / f}$ female mice were intraperitoneally injected with $7.5 \mathrm{IU}$ of pregnant mare's serum gonadotropin (Sigma-Aldrich, St. Louis, MO, USA) to induce folliculogenesis, followed by injection of $7.5 \mathrm{IU}$ of human chorionic gonadotropin (Sigma-Aldrich) 48 hours later to induce superovulation. Cumulus-oocyte complexes were collected from the oviducts 13 to 14 hours post-injection. The metaphase II (MII) oocytes were retrieved in Quinn's Advantage Medium with HEPES (Sage In Vitro Fertilization, Trumbull, CT, USA) containing 20\% fetal bovine serum (FBS) (Gibco, Grand Island, NY, USA) at $37^{\circ} \mathrm{C}$. The cumulus cells were then removed by using $300 \mu \mathrm{g} / \mathrm{mL}$ hyaluronidase (Sigma-Aldrich).

Table 1. Primers used for genotyping and RT-PCR of $A \operatorname{tg} 7^{d / d}$ mice

\begin{tabular}{|c|c|c|c|}
\hline Gene name & Sequence $\left(5^{\prime}-3^{\prime}\right)$ & Product size $(b p)$ & GenBank accession No. \\
\hline \multicolumn{4}{|l|}{ Primer name } \\
\hline \multirow[t]{2}{*}{ Floxed Atg7 } & TGG CTG CTA CTT CTG CAA TGA TGT & Wildtype: 1,500 & [7] \\
\hline & CAG GAC AGA GAC CAT CAG CTC CAC & Floxed: 550 & \\
\hline \multirow[t]{2}{*}{ Zp3-Cre transgene } & GCG GTC TGG CAG TAA AAA CTATC & 100 & \\
\hline & GTG AAA CAG CATTGC TGT CACTT & & \\
\hline \multirow[t]{2}{*}{ Zp3-Cre positive } & CTA GGC CAC AGA ATT GAA AGA TCT & 324 & \\
\hline & GTA GGT GGA AATTCT AGC ATC ATC C & & \\
\hline \multicolumn{4}{|l|}{ Gene name } \\
\hline \multirow[t]{2}{*}{ Atg7 } & F ATG CCA GGA CAC CCT GTG AAC TTC & 350 & NM_028835.4 \\
\hline & R ACA TCA TTG CAG AAG TAG CAG CCA & & \\
\hline \multirow[t]{2}{*}{ rpl7 } & F TCA ATG GAG TAA GCC CAA AG & 246 & NM_011291.5 \\
\hline & R CAA GAG ACC GAG CAATCA AG & & \\
\hline \multirow[t]{2}{*}{ Cre } & F CATTTG GGC CAG CTA AAC AT & 454 & {$[10]$} \\
\hline & R CCC GGC AAA ACA GGT AGTTA & & \\
\hline
\end{tabular}

RT-PCR, reverse transcription polymerase chain reaction; $Z p 3$, zona pellucida protein 3; rpl7, ribosomal protein L7. 


\section{Vitrification and warming procedure}

The vitrification procedure that was used is described in [11]. The oocytes were pre-equilibrated with a solution of $7.5 \%$ ethylene glycol (EG) (Sigma-Aldrich), 7.5\% dimethyl sulfoxide (DMSO) (Sigma-Aldrich) and $20 \%$ FBS for 2.5 minutes. They were then equilibrated with $15 \%$ EG, 15\% DMSO, 20\% FBS, and 0.5 M sucrose (Fisher Scientific, Fair Lawn, NJ, USA) for 20 seconds. The equilibrated oocytes was loaded onto a Cryotop (Kitazato, Shizuoka, Japan) and immersed in liquid nitrogen $\left(\mathrm{LN}_{2}\right)$. The vitrified oocytes were stored in $\mathrm{LN}_{2}$ for 2 weeks. After 2 weeks, they were warmed with sucrose solutions of decreasing concentrations $(0.5,0.25,0.125$, and $0 \mathrm{M})$ and $20 \%$ FBS for $2.5 \mathrm{~min}$ utes each. The vitrified-warmed oocytes were then cultured for 1 to 2 hours in medium with HEPES containing $20 \%$ FBS for recovery.

\section{In vitro fertilization and embryo culture}

In vitro fertilization (IVF) was performed according to the procedure described by Cha et al. [16]. Epididymal sperm waom 10-11 weekold male ICR mice. For capacitation, a drop of sperm suspension was added to Quinn's Advantage Fertilization Medium human tubal fluid (HTF) (Sage) containing 10\% substitute protein serum (SPS, Sage), and incubated for 90 minutes at $37^{\circ} \mathrm{C}$ in $5 \% \mathrm{CO}_{2}$. For IVF, the capacitated sperm suspension was added to oocytes in HTF containing $10 \%$ SPS. After 6 hours, the oocytes were washed four times with HTF containing $10 \%$ SPS and finally with amino acid-supplemented potassium simplex optimized medium (KSOM-AA) medium. They were then cultured in KSOM-AA drops overnight. Fertilization was confirmed 24 hours later by observing the two-cell embryos. They were cultured for 5 days to assess embryonic development.

\section{RNA extraction and RT-PCR}

Total RNA from the MII oocytes (50 oocytes/sample) and ovaries were extracted using TRIzol Reagent (Invitrogen, Carlsbad, CA, USA) according to the manufacturer's protocol. Rabbit $a$-globin RNA (10 $\mathrm{pmol} / \mathrm{sample}$, Sigma-Aldrich) was used as an external control [11]. To isolate minute amounts of RNA from oocytes, $3 \mathrm{M}$ sodium acetate ( $\mathrm{pH}$ 5.2) and glycogen were used in a modified protocol. The resuspended RNA was treated with RNase-free DNase I (Roche, Mannheim, Germany) for 20 minutes at room temperature to remove any residual genomic DNA. Total RNA was then reverse transcribed using Moloney murine leukemia virus reverse transcriptase (BEAMS Biotechnology, Seongnam, Korea) and random hexamer primers (Roche) for cDNA synthesis. The cDNA samples were used as templates for PCR analysis. PCR was performed with Prime Taq Premix $(2 \times)$ (Genet Bio, Daejeon, Korea). Ribosomal protein L7 was used as an internal control. The RT-PCR was repeated four times with independent sample sets. The sequences of primers used for PCR analysis are given in Table 1.

\section{Immunofluorescence staining and confocal microscopy}

Oocytes were fixed and permeabilized with 4\% paraformaldehyde with $0.1 \%$ Triton X-100 in phosphate-buffered saline (PBS) for 20 minutes, and washed twice with $0.1 \%$ Triton X-100 in PBS for 5 minutes each. The oocytes were then blocked in a $2 \%$ bovine serum albumin (BSA)/PBS drop for 1 hour. They were then incubated with a primary antibody drop containing anti-LC3 (rabbit polyclonal, 1:400, Cell Signaling Technology, Danvers, MA, USA) in 2\% BSA/PBS overnight at $4^{\circ} \mathrm{C}$. The oocytes were washed three times in $2 \%$ BSA/PBS, and incubated with an Alexa Fluor 488 chicken anti-rabbit secondary antibody (1:250, Molecular Probes, Waltham, MA, USA) for 40 minutes. The oocytes were then counterstained with TO-PRO-3-iodide (1:250, Molecular Probes), washed in 2\% BSA/PBS, directly placed on a glass slide, and covered with a glass coverslip that was sealed with transparent nail polish. Rabbit lgG was used as a mock control. Immunofluorescence images were obtained using an LSM710 confocal microscope (Carl Zeiss, Oberkochen, Germany), and analyzed using the Zen 2009 Light Edition (Carl Zeiss), a platform associated with the confocal microscope. For quantification of the LC3 puncta, each oocyte was imaged at four planes ( $3 \mu \mathrm{m}$ intervals) using an LSM710 confocal microscope. The number of LC3-positive puncta was quantified by using the count tool in Image J ver. 1.50b (National Institutes of Health, Bethesda, ME, USA, http://imagej.nih.gov/ij/).

\section{Statistical analyses}

All data were graphed using GraphPad Prism ver. 5 (GraphPad, La Jolla, CA, USA). Statistical analyses were performed using the Student $t$-test (one-tailed). The criteria for statistical significance were $p<0.05$ and $p<0.001$.

\section{Results}

\section{Confirmation of Atg7 deletion in oocytes from Atg $^{f / f} ; \mathrm{Zp3}-$ Cre mice}

To examine whether defective autophagy affects the survival, fertilization, and development of vitrified-warmed mouse oocytes, we used Atg7 floxed mice [7] bred with Zp3-Cre transgenic mice [10]. At-

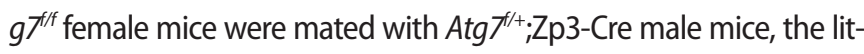
ters of which were selected as experimental (Atg ${ }^{f / f} ; Z \mathrm{p} 3-C r e$, referred hereafter as $A \operatorname{tg} 7^{d / d}$ ) or control (Atg $7^{f / /}$ ) by genotyping (Figure 1A). Deletion of the Atg7 gene in Atg ${ }^{d / d}$ MIl oocytes was confirmed by absence of Atg7 mRNA expression in MIl oocytes in the RT-PCR analysis. As shown in Figure 1B, Atg7 mRNA expression was maintained in the entire ovary of $A t g 7^{d / d}$ mice whereas their oocytes completely lost their expression. As expected, Cre mRNA expression was only noted in the Mll oocytes of $A t g 7^{d / d}$ mice. This suggests a complete deletion of Atg7 in the oocytes specifically. 
A

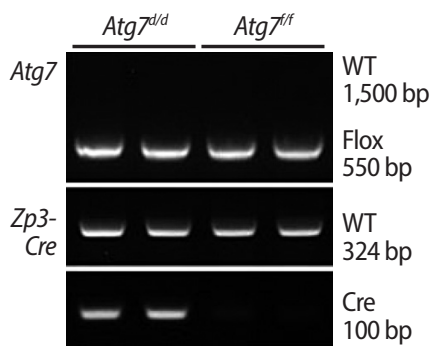

B

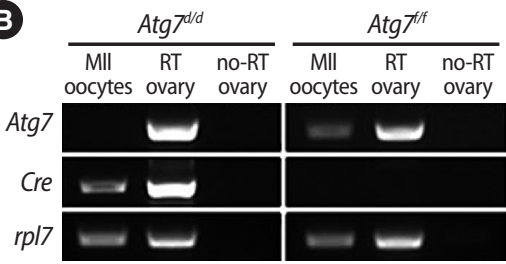

C

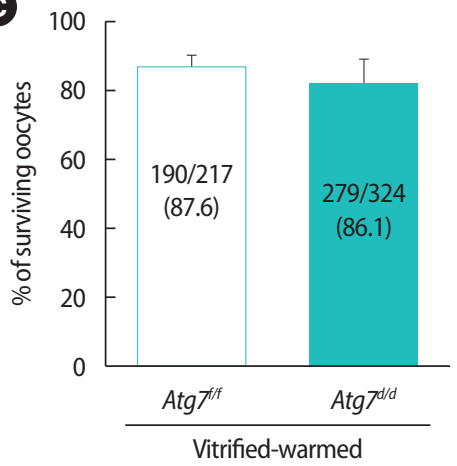

D
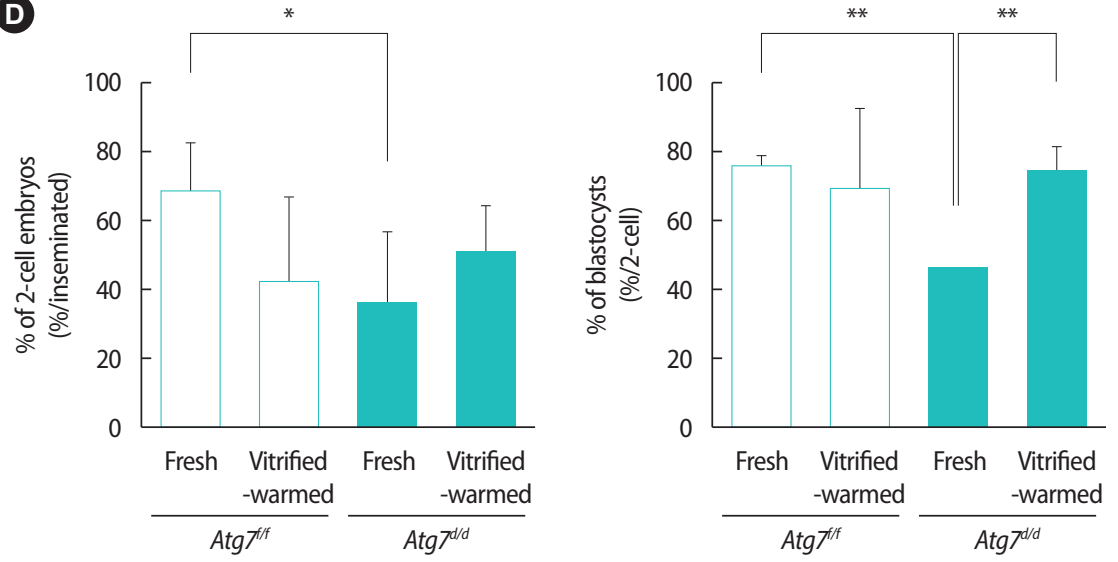

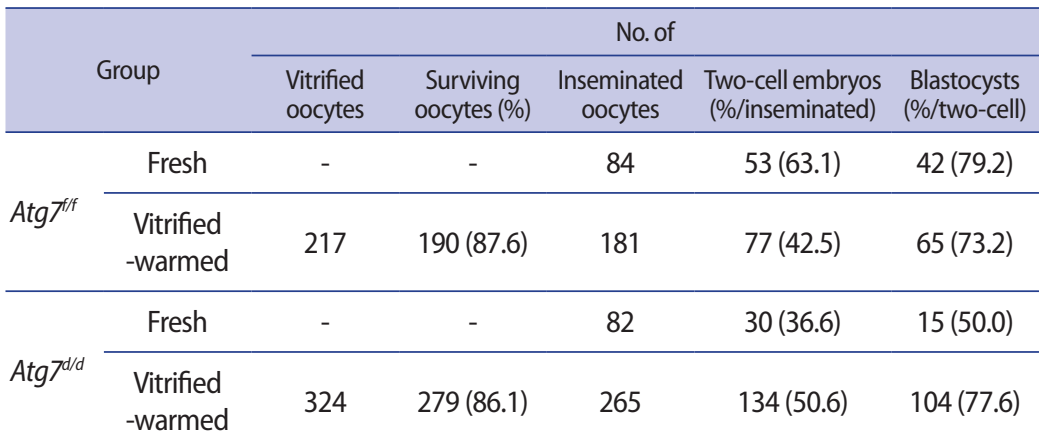

Figure 1. Survival, fertilization, and development of vitrified-warmed oocytes from Atg $7^{f f} ; \mathrm{Zp} 3-C r e$ mice. (A) Oocyte-specific deletion of the Atg7 gene in Atg $f^{(f / F} ; Z$ p3-Cre mice (Atg ${ }^{d / d}$ ) was confirmed. Primers used for genotyping are shown in Table 1. (B) RT-PCR results of Atg7 and Cre in oocytes from Atg $7^{d / d}$ and Atg $7^{f / f}$ mice. RNA from the ovaries (not reverse transcribed) was used as a negative control. rpl7 expression was examined as the loading control. Primers used for RT-PCR are shown in Table 1. (C) Survival rates of Atg $7^{d / d}$ and Atg $7^{f / f}$ oocytes after vitrification and warming. There was no significant difference in the survival rates of the Atg7 deficient oocytes $(p=0.500)$. The values represent the mean \pm standard deviation $(n=4)$. Statistical significance was assessed by the Student $t$-test. (D) Fertilization and developmental rates of vitrified-warmed Atg $7^{d / d}$ and Atg $7^{f / f}$ oocytes. The values represent the mean \pm standard deviation $(n=3-4)$. The table shows the total number and percentage of oocytes that were used in the barograms. Statistical significance was assessed by the Student $t$-test. Zp3, zona pellucida protein 3; rpl7, ribosomal protein L7; RT-PCR, reverse transcription polymerase chain reaction; RT, reverse transcription; no-RT, no reverse transcription. ${ }^{*} p<0.05 ;{ }^{* *} p<0.001$.

\section{Survival of oocytes from $A \operatorname{tg} 7^{d / d}$ mice after vitrification}

The survival rates of MIl oocytes after vitrification and warming in $A \operatorname{tg} 7^{\mathrm{d} / \mathrm{d}}$ and $A \operatorname{tg} \mathrm{T}^{\mathrm{fff}}$ mice were evaluated (Figure 1C) and found to be similar (Atg ${ }^{f f f}:$ Atg $\left.{ }^{\mathrm{d} / d}=87.6 \%: 86.1 \%, p=0.500\right)$. This indicates that the absence of autophagic activation in vitrified-warmed oocyte does not adversely affect oocyte survival after vitrification.

\section{Fertilization and developmental rates of oocytes from $A \operatorname{tg} 7^{d / d}$ mice after vitrification-warming}

The surviving $A \operatorname{tg} 7^{f / f}$ and $A \operatorname{tg} 7^{d / d}$ oocytes were inseminated, and their fertilization and developmental rates were assessed. As shown in Figure 1D, fresh oocytes from the Atg $7^{d / d}$ mice showed significantly lower rates of fertilization (Atg ${ }^{\mathrm{t} / \mathrm{f}} \mathrm{Atg} \mathrm{At}^{\mathrm{d} / \mathrm{d}}=63.1 \%: 36.6 \%, p=0.047$ ) and development (Atg ${ }^{\mathrm{t} / \mathrm{f}}$ :Atg $\left.\mathrm{J}^{\mathrm{d} / \mathrm{d}}=79.2 \%: 50.0 \%, p<0.001\right)$ compared with the control oocytes. This is in concordance with a previous study where Atg5-deficient oocytes showed reduced rates of embryonic development [17]. In the vitrified-warmed groups, the fertilization rate was comparable between the two groups (Atg ${ }^{f f /}: A \operatorname{Atg}^{\mathrm{d} / d}=42.5 \%: 50.6 \%$, $p=0.289$ ). Interestingly, vitrified-warmed Atg $7^{d / d}$ oocytes showed a higher developmental rate to the blastocyst stage than fresh Atg $7^{d / d}$ oocytes (fresh:vitrified-warmed $=50.00 \%: 77.6 \%, p<0.001$ ).

\section{Expression of LC3 in Atg $7^{d / d}$ oocytes}

Among the many molecules involved in the autophagy pathway, LC3 is widely used to monitor dynamic autophagic flux by western blotting, immunofluorescence staining, or live imaging [1]. In our 
A

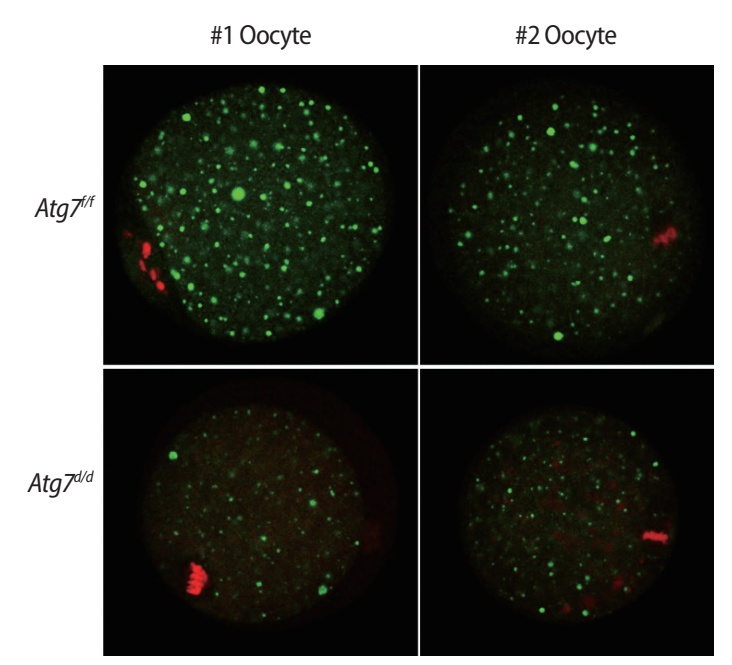

B

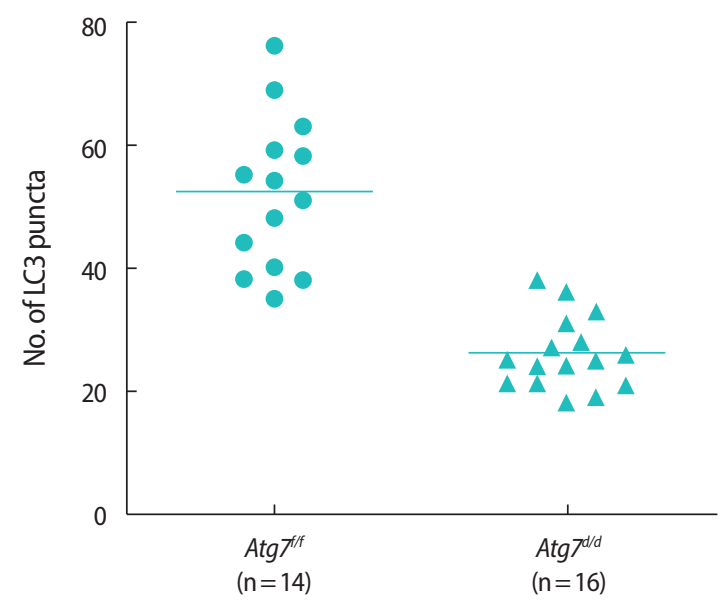

Figure 2. Immunofluorescence staining of LC3 in Atg $7^{\text {fff }} \mathrm{Mll}$ oocyte. (A) Two representative oocytes are shown (\#1 and \#2). MIl oocytes from At$g^{t / f}$ and $A \operatorname{tg} 7^{d / d}$ mice were vitrified-warmed and subjected to immunofluorescence staining with the anti-LC3 antibody. Alexa Fluor 488 (Molecular Probes, Waltham, MA, USA)-conjugated chicken anti-rabbit lgG was used as the secondary antibody (green), and nuclei were counterstained with TO-PRO-3-iodide (Molecular Probes) (red). Images were obtained by confocal microscopy (shown at $\times 80$ ). (B) For each oocyte, four images were captured at $3 \mu \mathrm{m}$ intervals, and green dots were counted by Image J software (National Institutes of Health, Bethesda, ME, USA). The number of green dots read in four planes was added and plotted by using GraphPad Prism (ver. 5, GraphPad, La Jolla, CA, USA) software. The horizontal lines represent the mean numbers. LC3, light chain 3; MII, metaphase II.

previous work showing activation of autophagy in oocytes during warming [11], we used GFP-LC3 transgenic oocytes, which enable observation of GFP-LC3 puncta formation by live imaging [18]. This method provides a consistent result when observing in vivo LC3 flux. Western blotting also provides a reliable readout of autophagic flux, as the conversion from the I-form to the II-form is easily distinguishable due to differential migrations [1]. On the other hand, observation by LC3 immunofluorescence staining requires caution [19]. While LC3-II proteins generally exhibit large puncta around autophagosomes, both LC3-I and LC3-II can be incorporated into protein aggregates in the cytoplasm [19], leading to misinterpretation under certain experimental conditions. Thus, we examined whether LC3 immunofluorescence staining can be reliably used as a monitoring method of autophagy in mouse oocytes using Atg $7^{\mathrm{d} / d}$ oocytes. We examined LC3 localization with the anti-LC3 antibody by immunofluorescence staining. As shown in Figure 2A, large LC3 puncta were notable in Atg f/f oocytes. Puncta were also noted in Atg $7^{\text {d/d }}$ oocytes, but the size and number were notably lower. For each oocyte, we counted the number of LC3-positive puncta and plotted them as shown in Figure 2B. The much lower number of puncta in the $A \operatorname{tg} \mathrm{J}^{\mathrm{d} / \mathrm{d}}$ oocytes suggests that $\mathrm{LC} 3$ immunostaining is a dependable monitoring method of autophagy in oocytes. Of note, a background level of LC3-positive signals is expected in Atg7 deficient tissues and cells and should therefore be interpreted with caution.

\section{Discussion}

Autophagy, a subcellular self-eating process, occurs in normal physiology and various disease conditions. The autophagic on-rate can increase by diverse insults and stimuli to cells, and the list of autophagic inducers is ever expanding [2]. Unveiling new inducers sheds light on the underlying regulatory mechanisms. In mammalian oocytes and embryos, several inducers of autophagy have been identified, mostly the adverse effectors [14]. Vitrification involves rapid temperature change that would affect the configuration of macromolecules, and autophagy is induced in mouse oocytes during recovery after vitrification [11]. In this work, we further show that Atg 7 is dispensable for cold stress exposed to oocytes during vitrification. Consistent with a study showing the role for autophagy in preimplantation embryonic development, as shown with Atg5 deficient oocytes [17], Atg $7^{d / d}$ oocytes also exhibit reduced developmental rates. However, reduced fertilization rates were not reported in the case of Atg5-deficient oocytes [17], suggesting that Atg7 may serve a more expanded role in the same pathway. With respect to vitrification, we observed that rates of survival, fertilization, and development were not further affected by it (Figure 1D). Thus, as we assumed in our previous work, autophagic induction during warming is likely a natural response to cold stress. Interestingly, vitrified-warmed At$g 7^{d / d}$ MIl oocytes seemed to develop better to the blastocyst stage (Figure 1D). While this observation is interesting, it remains elusive 
what brought about such an outcome, which warrants further investigation. We recently showed that rapamycin treatment of MII oocytes increased autophagy and had an adverse effect on fertilization and development [20]. Taken together with our findings herein, suppressed autophagy may have a beneficial effect on the development of vitrified-warmed oocytes.

Using Atg $7^{d / d}$ oocytes as a negative control, we established that LC3 immunofluorescence staining can be reliably used to monitor autophagy in oocytes. While small LC3 puncta were observed in Atg $7^{d / d}$ oocytes at the background level, the number of large LC3 puncta was significantly higher in Atg ${ }^{f f f}$ oocytes (Figure 2), making it possible to distinguish autophagic activation by this method.

In conclusion, we propose that mouse oocytes express the Atg7 gene, and this gene is required for normal fertilization and development to the blastocyst stage but not necessary for a vitrificationwarming-induced response that is autophagic.

\section{Conflict of interest}

No potential conflict of interest relevant to this article was reported.

\section{Acknowledgments}

The authors would like to thank the members of the laboratory for their constant support.

\section{References}

1. Klionsky DJ, Abdalla FC, Abeliovich $\mathrm{H}$, Abraham RT, AcevedoArozena A, Adeli K, et al. Guidelines for the use and interpretation of assays for monitoring autophagy. Autophagy 2012;8:445-544.

2. Boya P, Reggiori F, Codogno P. Emerging regulation and functions of autophagy. Nat Cell Biol 2013;15:713-20.

3. Yang Z, Klionsky DJ. Eaten alive: a history of macroautophagy. Nat Cell Biol 2010;12:814-22.

4. Choi S, Shin H, Song H, Lim HJ. Suppression of autophagic activation in the mouse uterus by estrogen and progesterone. J Endocrinol 2014;221:39-50.

5. Ohsumi Y. Molecular dissection of autophagy: two ubiquitin-like systems. Nat Rev Mol Cell Biol 2001;2:211-6.

6. Ichimura Y, Kirisako T, Takao T, Satomi Y, Shimonishi Y, Ishihara N, et al. A ubiquitin-like system mediates protein lipidation. Nature 2000;408:488-92.

7. Komatsu M, Waguri S, Ueno T, Iwata J, Murata S, Tanida I, et al.
Impairment of starvation-induced and constitutive autophagy in Atg7-deficient mice. J Cell Biol 2005;169:425-34.

8. Hale AN, Ledbetter DJ, Gawriluk TR, Rucker EB 3rd. Autophagy: regulation and role in development. Autophagy 2013;9:951-72.

9. Epifano O, Liang LF, Familari M, Moos MC Jr, Dean J. Coordinate expression of the three zona pellucida genes during mouse oogenesis. Development 1995;121:1947-56.

10. de Vries WN, Binns LT, Fancher KS, Dean J, Moore R, Kemler R, et al. Expression of Cre recombinase in mouse oocytes: a means to study maternal effect genes. Genesis 2000;26:110-2.

11. Bang S, Shin H, Song H, Suh CS, Lim HJ. Autophagic activation in vitrified-warmed mouse oocytes. Reproduction 2014;148:11-9.

12. Martinez-Burgos M, Herrero L, Megias D, Salvanes R, Montoya $M C$, Cobo AC, et al. Vitrification versus slow freezing of oocytes: effects on morphologic appearance, meiotic spindle configuration, and DNA damage. Fertil Steril 2011;95:374-7.

13. Gualtieri R, Mollo V, Barbato V, Fiorentino I, laccarino M, Talevi R. Ultrastructure and intracellular calcium response during activation in vitrified and slow-frozen human oocytes. Hum Reprod 2011;26:2452-60.

14. Lim HJ, Song H. Evolving tales of autophagy in early reproductive events. Int J Dev Biol 2014;58:183-7.

15. Practice Committees of American Society for Reproductive Medicine; Society for Assisted Reproductive Technology. Mature oocyte cryopreservation: a guideline. Fertil Steril 2013;99:37-43.

16. Cha SK, Kim BY, Kim MK, Kim YS, Lee WS, Yoon TK, et al. Effects of various combinations of cryoprotectants and cooling speed on the survival and further development of mouse oocytes after vitrification. Clin Exp Reprod Med 2011;38:24-30.

17. Tsukamoto S, Kuma A, Murakami M, Kishi C, Yamamoto A, Mizushima N. Autophagy is essential for preimplantation development of mouse embryos. Science 2008;321:117-20.

18. Mizushima N, Yamamoto A, Matsui M, Yoshimori T, Ohsumi Y. In vivo analysis of autophagy in response to nutrient starvation using transgenic mice expressing a fluorescent autophagosome marker. Mol Biol Cell 2004;15:1101-11.

19. Kuma A, Matsui M, Mizushima N. LC3, an autophagosome marker, can be incorporated into protein aggregates independent of autophagy: caution in the interpretation of LC3 localization. Autophagy 2007;3:323-8.

20. Lee GK, Shin H, Lim HJ. Rapamycin influences the efficiency of in vitro fertilization and development in the mouse: a role for autophagic activation. Asian-Australas J Anim Sci 2015 Oct 8 [Epub]. http://dx.doi.org/10.5713/ajas.15.0762. 